\title{
High Speed, High Resolution and Large Area X-ray Imaging Using Silicon Drift Detectors
}

\author{
R. Terborg*, J. Berlin*, T. Salge* \\ *Bruker Nano GmbH, Schwarzschildstr. 12, 12489 Berlin, Germany
}

Silicon Drift Detectors (SDDs) have become state-of-the-art in energy dispersive spectrometry (EDS) on scanning electron microscopes (SEMs), transmission electron microscopes (S/TEMs) and electron microprobes (EMPs), outperforming $\mathrm{Si}(\mathrm{Li})$ detectors in almost every aspect. SDDs have an excellent energy resolution (down to $123 \mathrm{eV}$ for $\mathrm{Mn}-\mathrm{K} \alpha$ ) and a high count rate capability. This allows low dwell times per pixel, making SDDs an ideal device for high speed and high resolution X-ray imaging [1] (e.g., Fig. 1). Combined with professional software and implemented stage control, X-ray imaging of large areas and complete samples is possible.

Position-tagged spectrometry (PTS), where a complete spectrum is stored for each pixel, permits data mining for specific elements. Acquisition times are reasonable even for samples that contain elements with strongly overlapping lines, because the quality of the spectra and the good energy resolution of SDDs allow small regions of interest to display the element distribution by intensity map and deconvolution of overlapping lines. Software tools further permit the analysis of certain areas or line scans within the PTS datacube, Figs. 2 and 3.

There is an increasing demand for analyses at high spatial resolution, for which low accelerating voltages are needed, and for investigations of sensitive samples, which involve low beam currents and low X-ray yields. On the other hand there is also a demand for even faster imaging. This can be fulfilled by improved X-ray collection efficiency and with even higher pulse throughput capabilities. Because of the design and stability of SDDs, system containing up to four detectors on a microscope can be used. This multiplies the throughput by parallel processing and increases the solid angle. Another benefit for spectrum imaging is the reduction of shadows when analyzing rough surfaces.

A new development is the four-channel detector: four SDDs integrated on one chip in one detection unit [2,3]. It provides a large solid angle and a high count rate capability, but has the advantage of a compact design. Four-channel detectors achieve output count rates above 1,000,000 cps and reduce pile-up and dead time effects. A special development is the annular four-channel detector, which is positioned between the microscope pole piece and the sample [3]. It offers a solid angle above $1 \mathrm{sr}$, which is up to 100 times larger than for conventional SDDs, and it reduces shadowing effects as well. It is therefore predestined for ultra fast X-ray imaging of sensitive samples at low beam currents and low accelerating voltages (e.g., Fig. 4).

Acknowledgements:

We thank ODP, P. Schulte, A. Deutsch, A. Kearsley and D. Pereira for providing samples.

References

[1] D.E. Newbury, Microsc. Microanal. 12 (Suppl. 2) (2006) 818.

[2] H. Soltau et al., Microsc. Microanal. 15 (Suppl. 2) (2009) 204.

[3] R. Terborg, A. Käppel, T. Salge, Microsc. Microanal. 16 (Suppl. 2) (2010) 1302. 


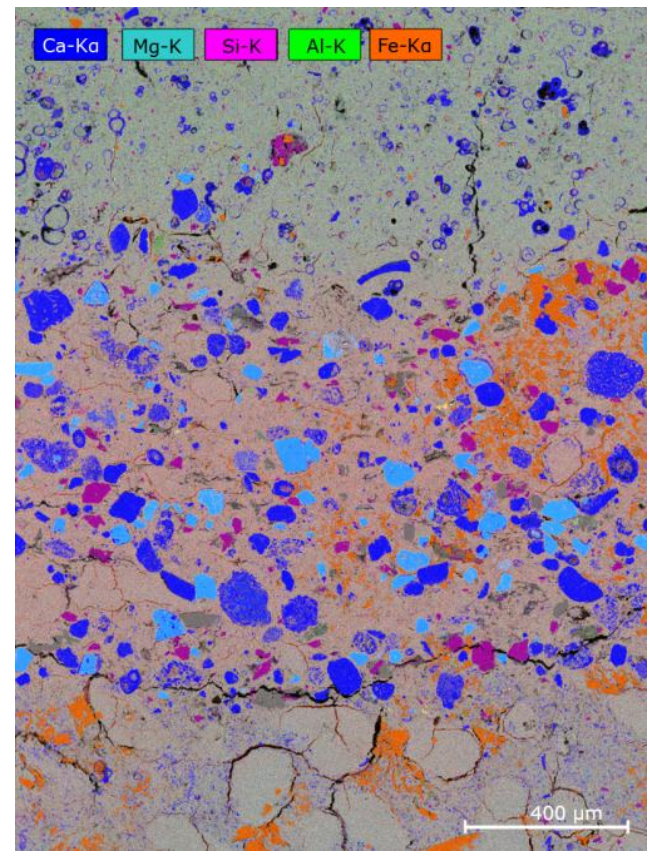

FIG. 1. High resolution element map of an oceanic drill core with ejecta from the Chicxulub impact $(3072 \times 4096$ pixel, $\sim 0.5 \mu \mathrm{m} / \mathrm{pixel}, 500 \mathrm{kcps}, 33 \mathrm{~min})$.
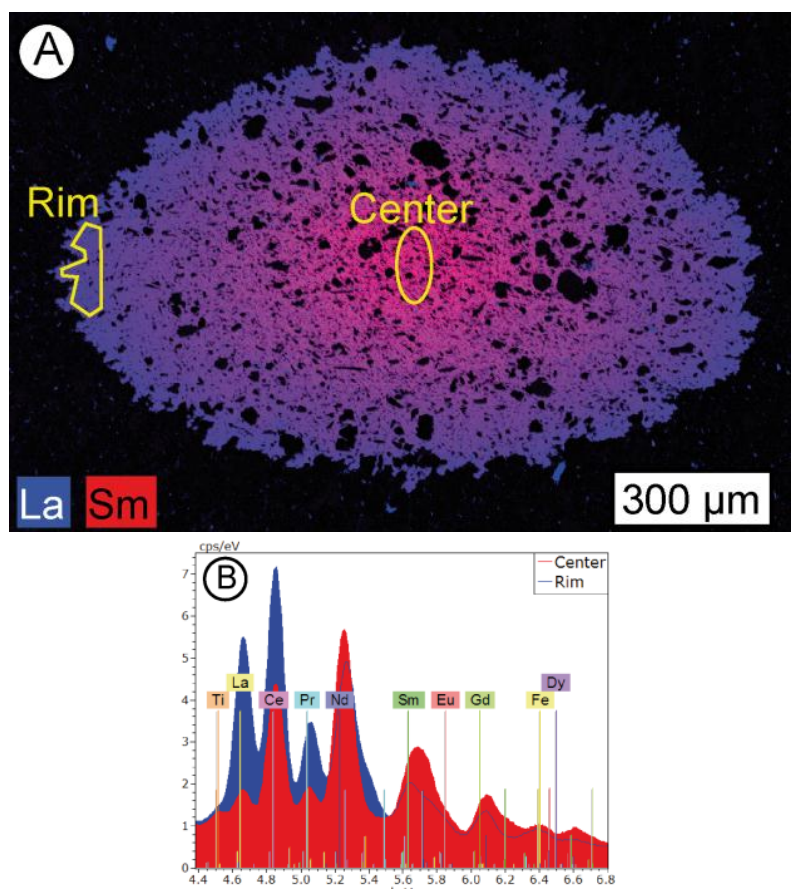

FIG. 2. (A) Intensity map of a zoned monazite and (B) area spectra extracted from the PTS datacube displaying zonations of REE with enrichment of La, $\mathrm{Ce}$ and depletion of $\mathrm{Nd}, \mathrm{Sm}, \mathrm{Gd}$ at the rim when compared to the center.
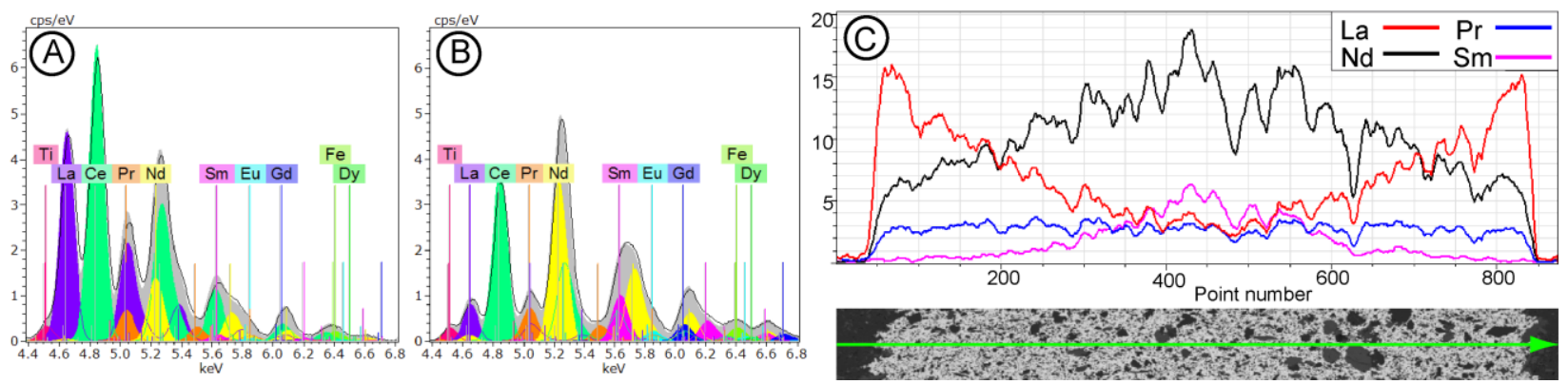

FIG. 3. Deconvolution results for the background-corrected spectrum of (A) rim and (B) center shown in Fig. 2B. Colored peaks are deconvolved peaks for each element. The grey area is the sum of all colored peaks and perfectly matches the acquired spectra (black line). (C) Quantitative line scan $(w t \%)$ showing the distribution of selected REE.
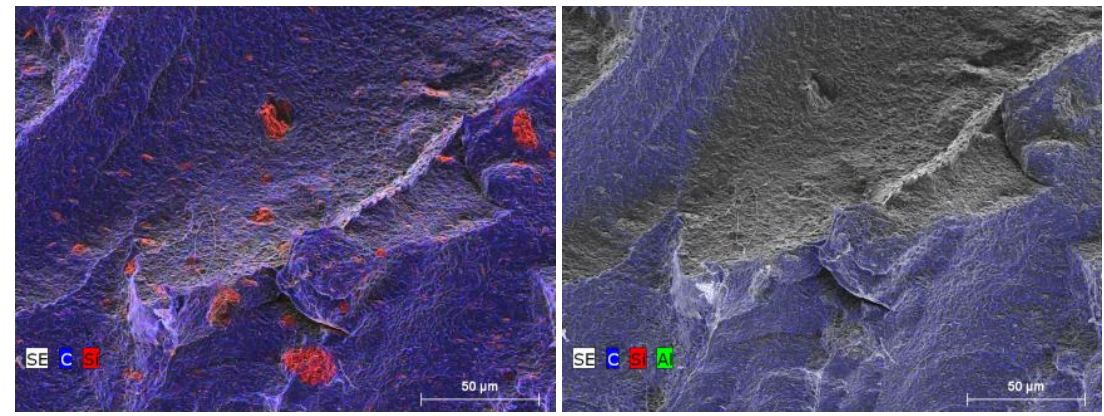

FIG. 4. X-ray image of a polymer composite containing nano organoclay ( $\mathrm{Si}, \mathrm{red})$. Left: annular four-channel detector.

Right: $30 \mathrm{~mm}^{2}$ SDD. 